Vehicle System Dynamics, Vol.25 Suppl., 1996, pp.413-425

\title{
Road Friction Coefficient Estimation For Vehicle Path Prediction
}

\author{
CHIA-SHANG LIU ${ }^{1}$ AND HUEI PENG ${ }^{2}$
}

\section{INTRODUCTION}

One of the most noticeable trends of the automotive industry in the 1990's is the emerging of active safety technologies. Instead of passive protection and crash worthiness, emphasis has been shifted toward accident prevention and damage reduction using active safety techniques. To develop effective active safety devices, it is necessary to estimate vehicle motions accurately, and reliable road friction estimation is one of the most important steps toward this goal.

This paper is part of an on-going research toward the development of a lane-departure warning system [1], one of the most technology-demanding active safety systems. The basic concept of the lane-departure warning system is to project vehicle trajectory, and compare with the perceived road geometry (obtained from vision systems) to calculate a performance metric termed "time to lane crossing" (TLC). When the calculated TLC is less than a threshold value, the control system will either issue warning signals or take intervention actions. To project the vehicle future trajectory accurately, we need to update vehicle parameters (speed, cornering stiffness, etc.) and estimate external disturbances (road super-elevation, wind gust, etc.). The estimation of external disturbances further depends on the vehicle parameters. Therefore, on-line estimation of road/tire characteristics is crucial for the TLC calculation and the overall lane-departure warning system. It is important to note that accurate road/tire friction estimation can also improve the performance of many other vehicle control/safety systems such as ABS, traction control, and 4WS systems.

Recently, various methods to identify the road friction coefficients have been developed. Dieckmann [2] developed a method which allows the exact measurement of wheel-slip in the order of $10^{-4}$. From the information of measured wheel slip the road surface variation is detected. Eichhorn and Roth [3] used optical and noise sensors at the front-end of the tire and stress and strain sensors inside the tire's tread and studied both "parameter-based" and "effect-based" road friction estimation methods. Ito et al. [4] uses the applied traction force and the resulting wheel speed difference between driven and non-driven wheels to estimate the road surface condition. Pal et al. [5] applied the neural-network based identification technique to predict the road frictional coefficient based on steady-state vehicle response signals. Pasterkamp and Pacejka [6] developed an on-line estimation method based on lateral force and self-aligning torque measurements. In this paper, we have developed a disturbance observer to identify the road surface friction coefficient. The effect of the road surface condition on vehicle path prediction (measured in TLC) is the main performance evaluation metric. The vehicle path prediction is obtained based on the 2-DOF lateral dynamic model (bicycle model). To calculate the TLC, the longitudinal tire force is first estimated

\footnotetext{
1 Graduate student

2 Assistant Professor, University of Michigan, Ann Arbor, MI 48109
} 
from a single wheel model. The road friction coefficient and the tire lateral force are then calculated based on an anisotropic tire model. Based on the estimated lateral force, the cornering stiffness is obtained. The updated cornering stiffness is then used in the bicycle model to compute the TLC. In the estimation scheme, the wheel speed and torque are assumed to be available (measured or calculated). From the measured wheel speed and the estimated vehicle velocity the tire slip ratio can be calculated. Vehicle velocity is derived by the non-driven wheel under driving conditions or estimated during braking conditions.

Two adaptation methods were used to estimate road surface conditions: least square method and a modified adaptive observer method. The least square method used in this paper is a recursive least square method with a forgetting factor [8]. The modified adaptive observer is a classical Lyapunov adaptive observer with a correcting term which improves the performance of the observer based on a linear relationship between the output and input signals. From simulation results we found that both methods can improve the TLC estimation accuracy noticeably under several cases.

\section{SYSTEM MODELS}

The tire models used to estimate the road surface condition consist of two parts: a single wheel rotational model and an anisotropic brush model. The model used for the vehicle path prediction and TLC calculations is a 2 DOF lateral dynamic model.

\subsection{Single Wheel Model}

The single wheel model shown in Fig. 1 is used to calculate the tire longitudinal force. The dynamics of the system in the state-space form is

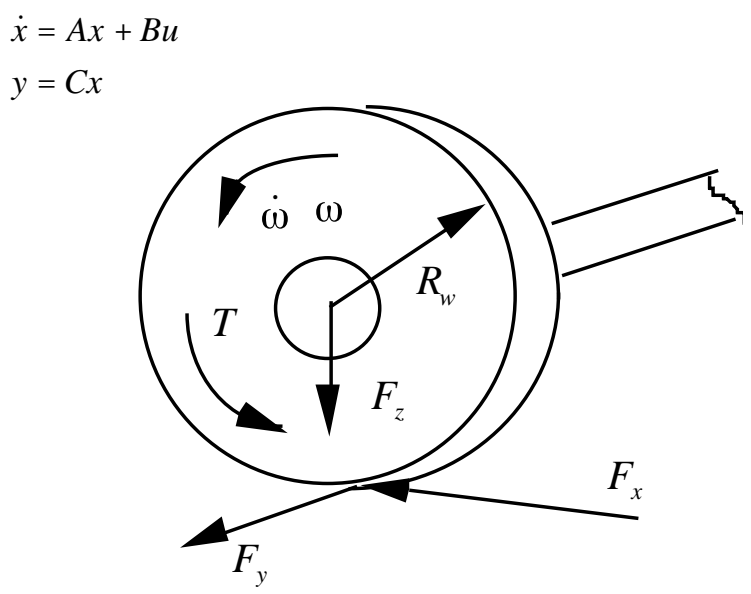

Fig. 1 Single Wheel Model

where $x$ is the wheel angular velocity $\omega, y$ is the measurement (which is also the wheel speed), $A=0, B=1$ and $C=1$. The external disturbance signal $u$ is

$$
u=\frac{T}{I_{w}}-F_{x} \frac{R_{w}}{I_{w}}
$$

where $T$ is the tire traction torque (negative when braking). $I_{w}$ is the wheel moment of inertia, $F_{x}$ is the tire longitudinal force and $R_{w}$ denotes the radius of the tire.

\subsection{Anisotropic Brush Model}

The anisotropic brush tire model represents the tire forces as functions of the tire slip ratio, slip angle and road surface condition. When the tire strain is not saturated, the longitudinal force $F_{x}$ and lateral force $F_{y}$ are 


$$
\begin{aligned}
& F_{x}=-\frac{k_{x} \sigma_{x}}{\sqrt{\left(k_{x} \sigma_{x}\right)^{2}+\left(k_{y} \sigma_{y}\right)^{2}}}\left\{3 c_{o} \sigma-3 \frac{1}{\mu} \frac{\left(c_{o} \sigma\right)^{2}}{F_{z}}+\frac{1}{\mu^{2}} \frac{\left(c_{o} \sigma\right)^{3}}{F_{z}^{2}}\right\} \\
& F_{y}=-\frac{k_{y} \sigma_{y}}{\sqrt{\left(k_{x} \sigma_{x}\right)^{2}+\left(k_{y} \sigma_{y}\right)^{2}}}\left\{3 c_{o} \sigma-3 \frac{1}{\mu} \frac{\left(c_{o} \sigma\right)^{2}}{F_{z}}+\frac{1}{\mu^{2}} \frac{\left(c_{o} \sigma\right)^{3}}{F_{z}^{2}}\right\}
\end{aligned}
$$

where $\sigma=\sqrt{\left(\sigma_{x}\right)^{2}+\left(\sigma_{y}\right)^{2}}$ is the total strain of the tire. $\sigma_{x}=-\frac{\kappa}{1+\kappa}$ and $\sigma_{y}=-\frac{\tan (\alpha)}{1+\kappa}$ are the strain components in the $\mathrm{x}$ and $\mathrm{y}$ directions, respectively. $k_{x}$ and $k_{y}$ are the tire horizontal stiffness, $\alpha$ is the tire slip angle, $\kappa$ is the tire slip ratio, and $F_{x}$ is the tire normal force. $\mu$ is the maximum traction coefficient.

The main reason we chose the brush model is because it is a easier model in mathematical manipulations. The parameters of the brush model were tuned to match the force curves of the magic formula[7] used in the simulation program. Fig 2 compares the tire forces from both the brush model and the magic formula under different road surface conditions and tire slip ratio. It can be seen that some discrepancies still exist. However, when the slip ratio is smaller than the peakforce value, these two models are quite close.

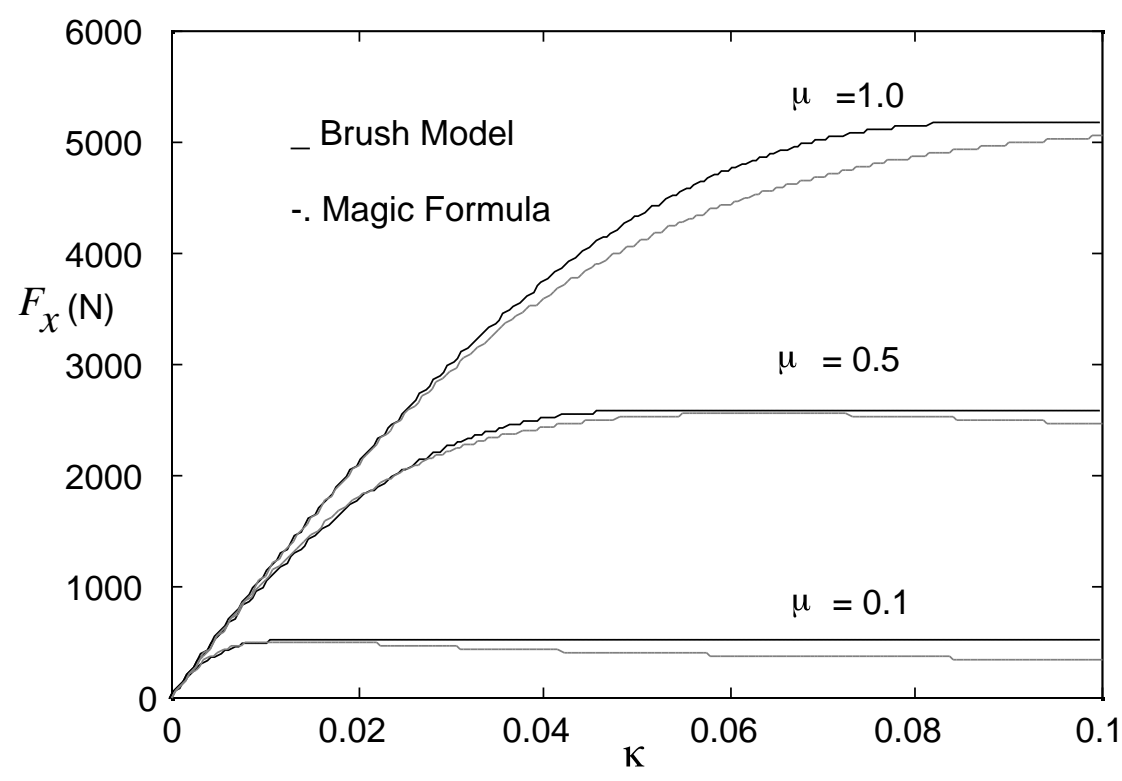

Fig.2 The Comparison between Brush Model and Magic Formula

\subsection{Vehicle Lateral Dynamics}

The 2 DOF bicycle model shown below is used for the TLC calculation:

$$
\left[\begin{array}{c}
\dot{\mathbf{v}} \\
\dot{\mathbf{r}}
\end{array}\right]=\left[\begin{array}{cc}
\frac{1}{m}\left(\frac{2 C_{s f}+2 C_{s r}}{\mathbf{u}}\right) & \frac{1}{m}\left(-m u+\frac{2 a C_{s f}-2 b C_{s r}}{\mathbf{u}}\right) \\
\frac{1}{I_{z}}\left(\frac{2 a C_{s f}-2 b C_{s r}}{\mathbf{u}}\right) & \frac{1}{I_{z}}\left(\frac{2 a^{2} C_{s f}+2 b^{2} C_{s r}}{\mathbf{u}}\right)
\end{array}\right]\left[\begin{array}{l}
\mathbf{v} \\
\mathbf{r}
\end{array}\right]+\left[\begin{array}{c}
\frac{-2 C_{s f}}{m} \\
\frac{-2 a C_{s f}}{I_{z}}
\end{array}\right] \delta_{f w}
$$

where $\mathbf{u}$ and $\mathbf{v}$ are the vehicle longitudinal and lateral speeds, $\mathbf{r}$ is the yaw rate, $m$ is the vehicle mass, and $I_{z}$ is the yaw moment of inertia. $C_{s f}$ and $C_{s r}$ are front and rear wheel cornering stiffness, respectively. $\delta_{f w}$ is the front wheel steering angle, and $a$ and $b$ are the distance from vehicle center of gravity to front and rear axles, respectively.

3. ESTIMATION SCHEMES 


\subsection{Recursive Least Square Method}

To estimate the longitudinal force from the single tire model, we can rewrite Eq.(1) into the discrete form as

$$
\frac{\omega(k+1)-\omega(k)}{\Delta t}-\frac{T(k)}{I_{w}}=\frac{-F_{x}(k) R_{w}}{I_{w}}
$$

where $\Delta t$ is the sampling time, and $k$ is the time index. For least-square estimation, we put the output signal in the following form

$$
y(k)=\Phi^{T}(k) \Theta(k)
$$

where for the single-wheel dynamics, we have $y(k)=\frac{\omega(k)-\omega(k-1)}{\Delta t}-\frac{T(k-1)}{I_{w}}, \Phi^{T}(k)=-\frac{R_{w}}{I_{w}}$ and $\Theta(k)=F_{x}(k)$

It is well-known that the recursive least-square estimation of the parameter vector $\hat{\Theta}(k)$ is

$$
\hat{\Theta}(k+1)=\hat{\Theta}(k)+\Psi(k+1) \Gamma(k) \Phi(k+1)\left[y(k+1)-\Phi^{T}(k+1) \hat{\Theta}(k)\right]
$$

and the two auxiliary matrices are updated in the following way

$$
\begin{aligned}
& \Gamma(k+1)=\frac{1}{\lambda}\left[\Gamma(k)-\Psi(k+1) \Gamma(k) \Phi(k+1) \Phi^{T}(k+1) \Gamma(k)\right] \\
& \Psi(k+1)=\frac{1}{\left[1+\Phi^{T}(k+1) \Gamma(k) \Phi(k+1)\right]}
\end{aligned}
$$

where $0<\lambda<1$ is the forgetting factor.

\subsection{Enhanced Adaptive Observer}

A standard Luenberger observer for the state-space model shown in Eq.(1) has the following form:

$$
\begin{aligned}
& \dot{\hat{x}}(t)=A \hat{x}(t)+B \hat{u}(t)+K(\hat{y}-y) \\
& \hat{y}(t)=C \hat{x}(t)
\end{aligned}
$$

where $K$ is the observer gain vector selected in a way such that the eigenvalues of the closed-loop state matrix $A+K C$ are located at desired locations. The error dynamics of this observer can be obtained by subtracting Eq.(1) from Eq.(8):

$$
\dot{e}=A_{k} e+B e_{u}
$$

where $e=\hat{x}-x, e_{u}=\hat{u}(t)-u(t)$ and $A_{k}=A+K C$. The updating law for the estimation of the unknown input is chosen to be

$$
\begin{aligned}
& \dot{\hat{u}}_{o}(t)=-B^{T} P e \\
& \hat{u}(t)=\hat{u}_{o}(t)-K_{o} e \\
& A_{k}^{T} P+P A_{k}=-Q
\end{aligned}
$$

where $\hat{u}_{o}(t)$ is the estimated input before correction, $\hat{u}(t)$ is the estimated input after correction, $K_{o}$ is the linear correction gain, and $P$ and $Q$ are positive definite matrices. The derivation of the updating law listed above is shown in the next section.

\subsection{Stability Analysis of the Enhanced Adaptive Observer}

To avoid any confusion, the derivation of the estimation scheme is divided into two steps: precorrection and after-correction. 
a) Pre-Correction

The observer of the system can be written as

$$
\dot{\hat{x}}=A \hat{x}+B \hat{u}_{o}+K(\hat{y}-y)
$$

where $\hat{u}_{o}(t)$ is the estimated input before correction. Subtract Eq.(1) form (13), we have

$$
\dot{e}=A_{k} e+B e_{\text {ио }}
$$

where $e_{u o}=\hat{u}_{o}(t)-u(t)$.

The Lyapunov candidate function is chosen as

$$
V=e^{T} P e+e_{u o}^{T} e_{u o}
$$

Taking the derivative of the Lyapunov function (15) along the trajectory of the error dynamics, we have

$$
\dot{V}=-e^{T} Q e+2 e^{T} P B e_{u o}+2 \dot{e}_{u o}^{T} e_{u o}
$$

Let

$$
\dot{e}_{u o}=-B^{T} P e
$$

then Eq.(17) becomes

$$
\dot{V}=-e^{T} Q e<0 .
$$

In other words, the updating law shown in Eq.(10) will guarantee the convergence of the estimation error for both state and input signals, for the cases when the unknown input $u(t)$ is constant. The aforementioned assumption of the update law imposes a limit on our ability to track time-varying functions. In the following, we will introduce a modification to reduce the effect of this drawback.

b) Modification of the Up-date law

We assumed that the estimated input error $e_{u o}$ is proportional to the state estimation error $e$ when $e$ is small, i.e.

$$
e_{u o}=K_{o} e
$$

In order to achieve the condition described in Eq.(18), Eq.(19) must be matched with Eq.(17). Take the derivative of Eq.(19) and substitute it into (17), we obtain the following relationship

$$
K_{o} A_{k}+K_{o} B K_{o}+B^{T} P=0
$$

From the above equation the proportional gain matrix $K_{o}$ can be solved. Substituting the obtained matrix $K_{o}$ into Eq.(19) and from the definition that $e_{u o}=\hat{u}_{o}(t)-u(t)$, we have the modified estimation signal

$$
\hat{u}(t)=\hat{u}_{o}(t)-e_{u o}
$$

Compare Eq.(19) with Eq.(21), Eq.(11) is obtained. The block diagram of the estimation scheme is shown in Fig. 3. 


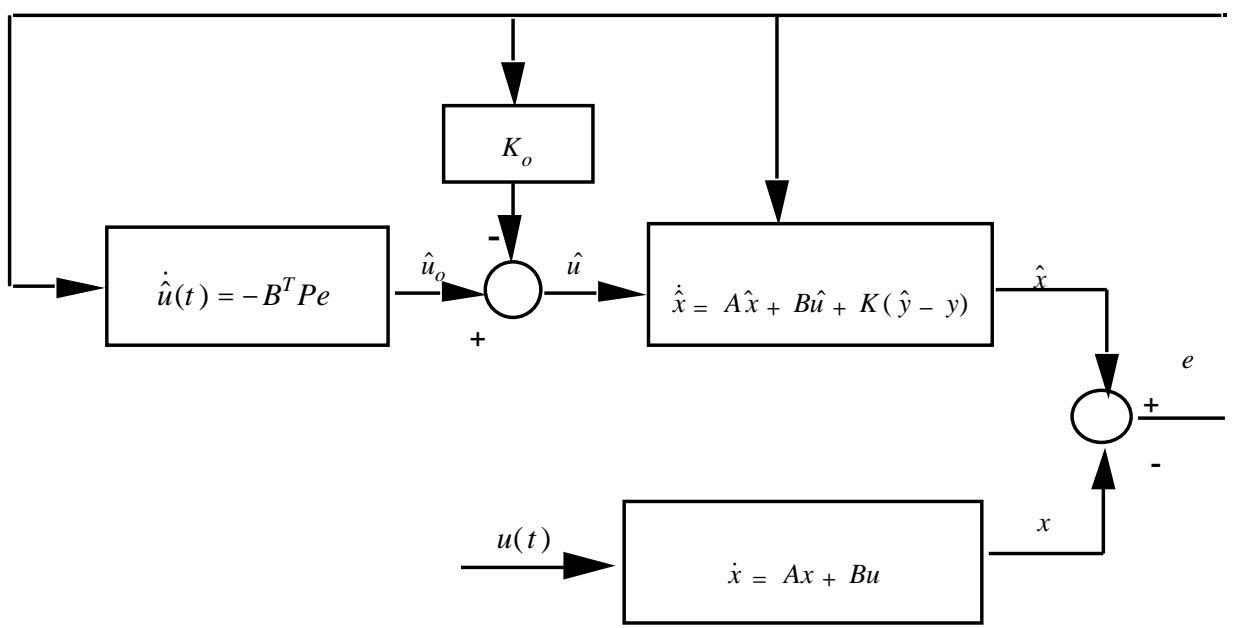

Fig.3 Schematic Representation of Estimation

\subsection{Estimation of Road Surface Condition}

The estimation of the road surface condition is based on the anisotropic brush model described in section 2.2. The idea is that it needs more tire slip to obtain the same tire-road interactive force when the road is slippery; thus road surface condition can be detected by observing tire shear force vs. tire slip. After estimating the tire longitudinal force, the tire-road friction coefficient $\mu$ can be obtained from (3a) and (3b):

$$
\begin{aligned}
& \hat{\mu}=\frac{a_{1}+\sqrt{a_{1}^{2}-4 a_{2} a_{0}}}{2 a_{2}} \quad \text { if } \quad \sigma \leq \sigma_{m} \\
& \hat{\mu}=\frac{\hat{F}}{F_{z}} \quad \text { if } \quad \sigma>\sigma_{m}
\end{aligned}
$$

where

$$
\begin{aligned}
& a_{0}=(\bar{\theta} \sigma)^{3} \\
& a_{1}=3(\bar{\theta} \sigma)^{2} F_{z} \\
& a_{2}=(3 \bar{\theta} \sigma-\hat{F}) F_{z}^{2} \\
& \bar{\theta}=\frac{4}{3} l_{1}^{2} l_{2} k \\
& \hat{F}=-\frac{\sqrt{\left(k_{x} \sigma_{x}\right)^{2}+\left(k_{y} \sigma_{y}\right)^{2}}}{\sigma_{x} k_{x}} \hat{F}_{x}
\end{aligned}
$$

$l_{1}, l_{2}$ and $k$ are tire-road patch length, width and stiffness, respectively. $l_{1}$ and $l_{2}$ depend on the tire pressure and $k$ varies with the tire slip ratio. $\sigma_{m}$ is the maximum total strain allowed on the tire.

\section{VEHICLE PATH PREDICTION AND TLC CALCULATION}

\subsection{Vehicle Path Prediction}

Vehicle path is determined by the complex interaction between human driver and the vehicle dynamics. To predict the future trajectory of the vehicle, it is a common practice [1] to assume that the steering angle is fixed and the vehicle speed remain constant in this future period of time. This assumption, albeit not true in many cases, will be used in most of the simulation cases in this paper. The prediction of the vehicle future path will be obtained from the bicycle model 
Vehicle System Dynamics, Vol.25 Suppl., 1996, pp.413-425

described in section 2.3. Since the relative motion of the car (with respect to the road) is of interest, the lateral dynamics described in (4) will be augmented to include curved-road scenarios.

a) Straight Road

When the road is straight, the following bicycle model is used:

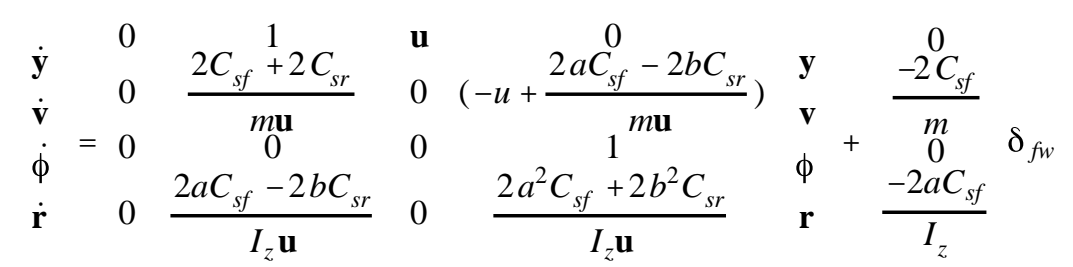

where $\mathrm{y}$ is the lateral displacement, $\mathrm{v}$ is the lateral speed, $\phi$ is the vehicle heading angle, and $\mathrm{r}$ is the yaw rate.

\section{b) Curved Road}

When the road is curved, the orientation of the road $\phi_{d}$ and the changing rate of the orientation $\mathbf{r}_{d}$ must be considered. Choosing $\dot{\mathbf{y}}$ and $\phi-\phi_{d}$ instead of $\mathbf{v}$ and $\phi$ as the state variables, the vehicle lateral dynamics in curved road will be

$$
\begin{aligned}
\frac{d}{d t}\left[\begin{array}{c}
\mathbf{y} \\
\dot{\mathbf{y}} \\
\phi-\phi_{d} \\
\mathbf{r}
\end{array}\right] & =\left[\begin{array}{llll}
0 & \frac{2 C_{s f}+2 C_{s r}}{m \mathbf{u}} & -\frac{2 C_{s f}+2 C_{s r}}{m} & \frac{2 a C_{s f}-2 b C_{s r}}{m \mathbf{u}} \\
0 & 0 & 0 & 1 \\
0 & \frac{2 a C_{s f}-2 b C_{s r}}{I_{z} \mathbf{u}} & -\frac{2 a C_{s f}-2 b C_{s r}}{I_{z}} & \frac{2 a^{2} C_{s f}+2 b^{2} C_{s r}}{I_{z} \mathbf{u}}
\end{array}\right]\left[\begin{array}{c}
\mathbf{y} \\
\dot{\mathbf{y}} \\
\phi-\phi_{d} \\
\mathbf{r}
\end{array}\right] \\
& +\left[\begin{array}{c}
0 \\
\frac{-2 C_{s f}}{m} \\
0 \\
\frac{-2 a C_{s f}}{I_{z}}
\end{array}\right] \delta_{f w}+\left[\begin{array}{c}
0 \\
-\mathbf{u} \\
-1 \\
0
\end{array}\right] \mathbf{r}_{d}
\end{aligned}
$$

\subsection{TLC Calculation}

TLC (time to lane crossing) is defined as the time for the center of gravity of the vehicle to cross the roadway edges. When calculating the TLC, the steering angle and the vehicle speed are assumed to be constant. TLC has been found to be a good metric for active safety measures. Warning, intervention or control actions can be taken based on the value of TLC. The TLC is calculated based on the lateral motion of the vehicle. More precisely, given the current lateral displacement from the lane edges, and assuming a constant steering input, TLC is defined as the time necessary for the lateral displacement to diminish to zero. It is obvious that the vehicle path prediction and TLC are affected by the tire cornering stiffness and steering input. On the other hand, tire lateral force needs to be calculated based on an estimated cornering stiffness. An initial guess of the tire cornering stiffness is chosen to estimate the vehicle lateral speed and the tire slip angle, the estimated vehicle lateral speed and tire slip angle are then used to calculate the updated tire cornering stiffens. Since the calculation of tire lateral speed and cornering stiffness depends on each other, we must solve them iteratively. Due to this iterative process, the estimation of the tire cornering stiffness is not fast enough to track the variation of the cornering stiffness if the real cornering stiffness varies dramatically.

\section{SIMULATION RESULTS}


The aforementioned methods were implemented on a simulation program which was developed by the CAPC team of the University of Michigan Transportation Research Institute (UMTRI). A 14 DOF vehicle model [11] was used, which includes the six degree of freedom of the sprung mass, and one degree of freedom for each of the four suspension linear motion and tire rotational motion. The simulation program utilizes the combined-slip magic formula model to compute the tire forces. In the simulations some variables are assumed to be measured. The wheel speed is measured, which is corrupted with $.1 \%$ random white noise. This level of accuracy was shown to be achievable under moderate vehicle speeds[12]. The high accuracy of the wheel speed measurement is necessary, since we need the high accurate of the tire slip ratio to identify the road surface condition. The measurement of yaw rate is contaminated by a $0.1 \%$ white noise error. The engine torque and braking torque have 5\% white noise error. The steering angle of the front wheel is assumed to be measured with a 0.1 degree white noise error.

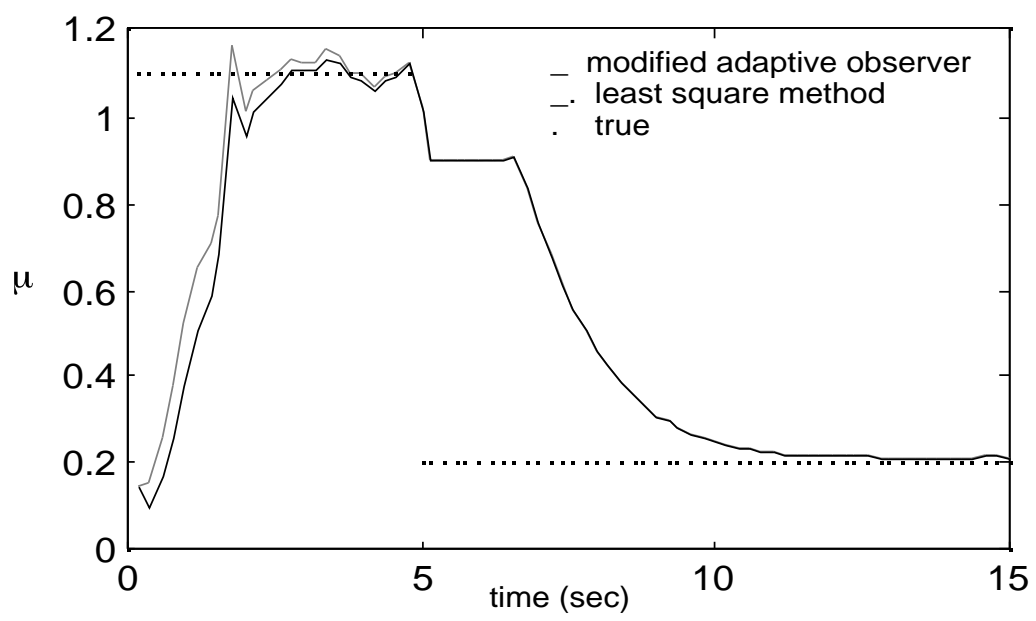

Fig. 4 Estimation of the Road Surface Condition

Both modified adaptive observer and least square algorithms are applied. It was found that both of these methods work well if designed properly. Fig. 4 shows the scenario when the road surface condition experienced a step-changes from $\mu=1.1$ to $\mu=0.2$. The driving torque is $440 \mathrm{~N}$ when $\mu=1.1$ and is reduced to $220 \mathrm{~N}$ when $\mu=0.2$. We can see that the fluctuation of the estimation result is large when $\mu$ is large. This is because it is difficult to distinguish $\mu$ when $\mu$ is large (see Figure 2).

Fig. 5 shows the estimated cornering stiffness for both front and rear wheels under three different road surface conditions. The steering wheel is assumed to be kept at 30 degree $(=1$ degree steering at tires). We can see that when $\mu=1.1$ and $\mu=0.5$ the cornering stiffness didn't deviate much from the initial guesses, but when $\mu=0.2$ the cornering stiffness will reduce to about 4000 $\mathrm{N} / \mathrm{rad}$. Since the cornering stiffness will not vary much when $\mu$ is larger than 0.5 , we will focus on the cases when the road surface is slippery. When $\mu$ is high, we would expect TLC to be very insensitive to the road surface condition. Fig. 6 shows the predicted path of the vehicle when the wheel steering angle is 20 degree $(=0.67$ degree steering at tires) and $\mu=0.1$. The results show that the TLC based on up-dated cornering stiffness is much more accurate. We also expect that the improvement from updated cornering stiffness will be larger when TLC is higher. In other words, it makes more sense to use updated cornering stiffness for warning, rather than on intervention and control actions. 
Vehicle System Dynamics, Vol.25 Suppl., 1996, pp.413-425

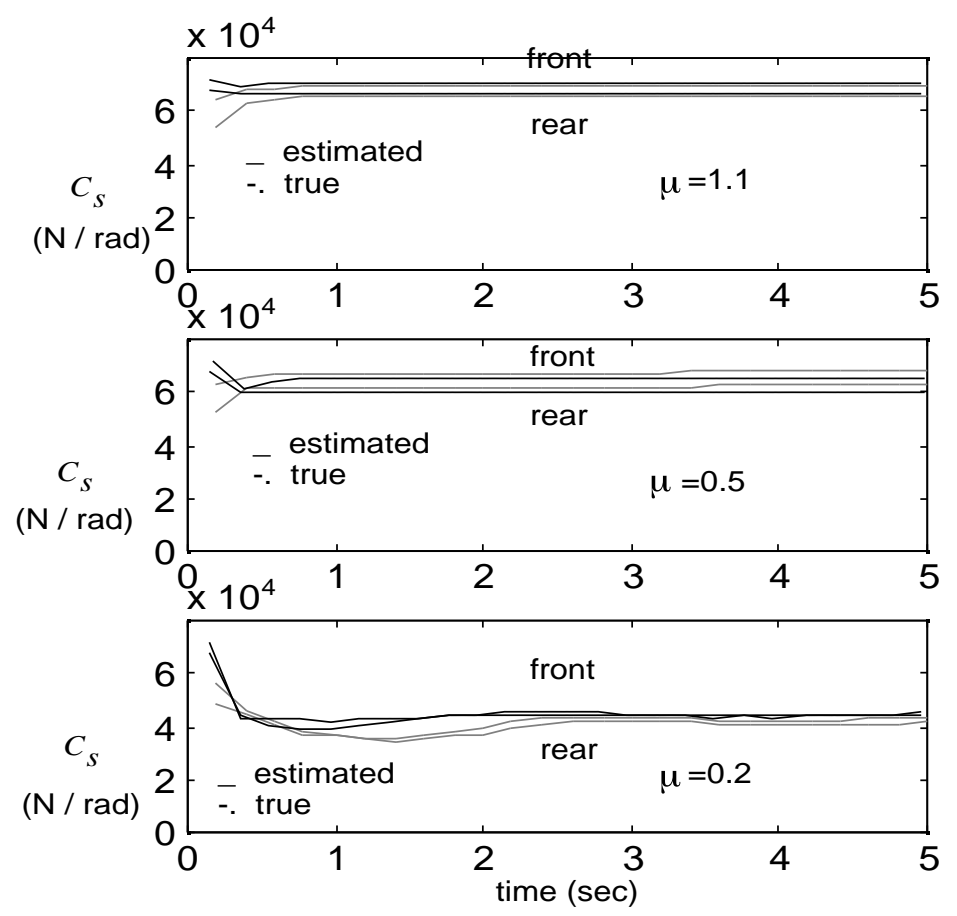

Fig. 5 Estimation of the Cornering Stiffness

In figure 7, the vehicle is assumed to have a 20 degree wheel steering angle at $18 \mathrm{~m} / \mathrm{sec}$. It is assumed that the road is straight and the distance between the c.g. of the vehicle and the road edge is $2 \mathrm{~m}$. The rms value of TLC estimation error is 0.02 second and 0.28 second for the updated and un-updated cases, respectively. Fig. 8 shows the calculated TLC under the same driving conditions except that the road has a radius of curvature $=1000 \mathrm{~m}$. The rms value of TLC estimation error is 0.187 second and 0.65 second for the updated and un-updated cases. It should be noted that the difference between the estimated TLC and the nominal TLC is larger in the in the curved-road case. The major reasons is that the TLC is larger in this case. Because the initial value of the cornering stiffness of the updated one is the same of that of the un-updated one, the initial calculated TLC will be the same in both cases.

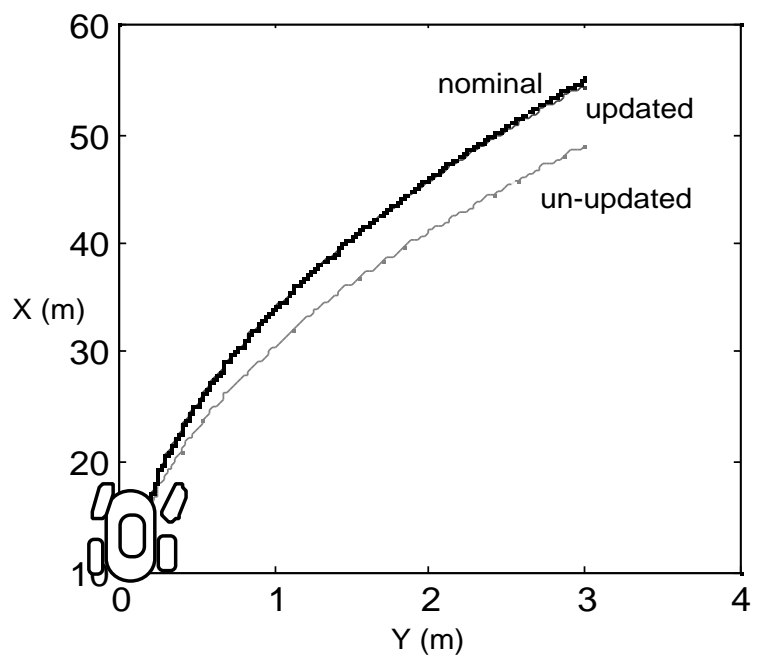

Fig. 6 Predicted Vehicle Path 


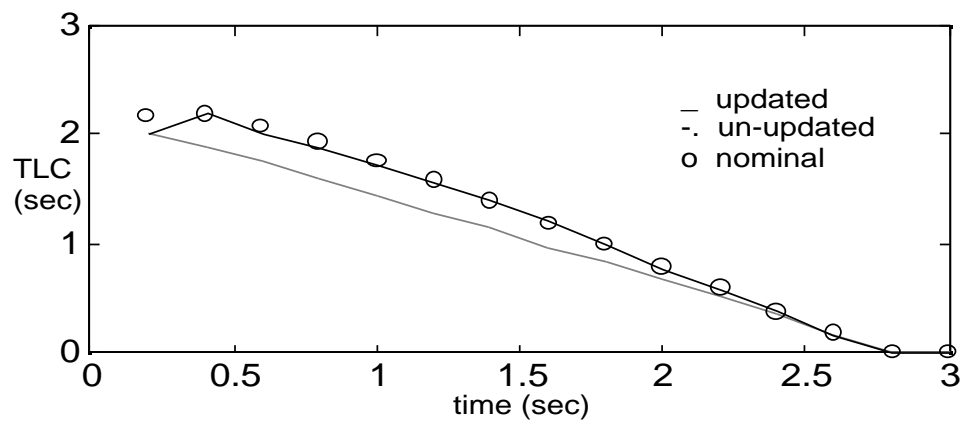

Fig. 7 Calculated TLC (Straight Road)

Fig. 9 shows the TLC of the vehicle with zero steering angle on a curved road. It can be seen that the 0.1 degree measurement noise of the front wheel steering angle has little effect on the TLC calculation. Only the results of the updated case is presented because the difference between the cornering stiffness of the updated and un-updated cases is small when tire slip angle is very small.

Fig. 10 shows the effect of vehicle load variation. A $200 \mathrm{~kg}$ luggage is assumed to be loaded in the trunk. Important parameters of the vehicle including c.g. location, vehicle mass and moment inertia are changed accordingly. The driving conditions and road geometry are the same as those of Fig. 8. The results show that the TLC based on updated information is still much more accurate than the un-updated case. The rms value of the estimation error is 0.09 second and $0 . .35$ second, respectively.

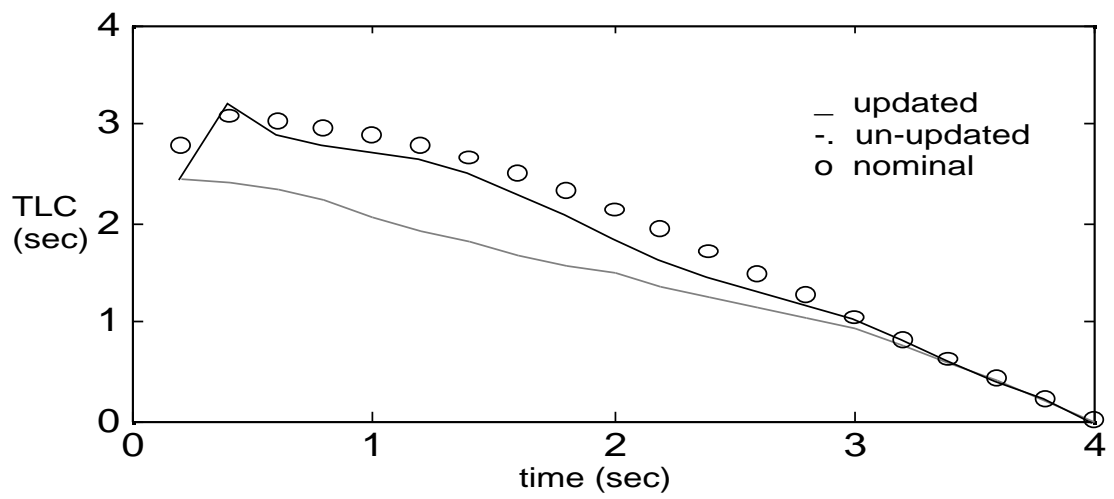

Fig. 8 Calculated TLC (Curved Road)

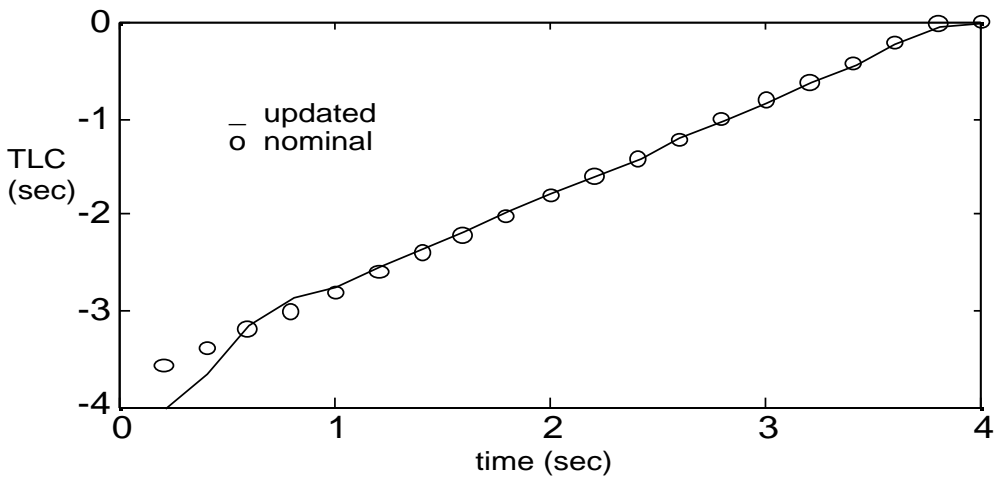

Fig. 9 Calculated TLC (curved road, zero steering angle) 


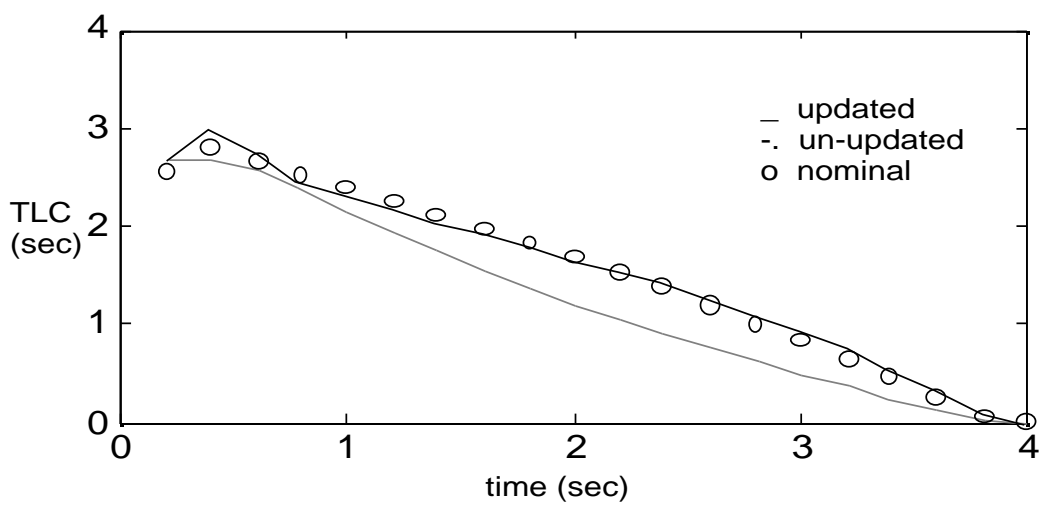

Fig. 10 Calculated TLC (curved road, extra load in trunk)

The effect of road gradient is shown in Fig. 11. The gradient of the road is assumed to be 0.05 $\operatorname{rad}($ about 3\%). All the other simulation parameters are the same as those of Figure 8. The rms value of TLC estimation error is 0.33 second and 0.78 second for the updated and un-updated cases, respectively. Under this circumstance, the identification schemes cannot provide satisfactory improvements.

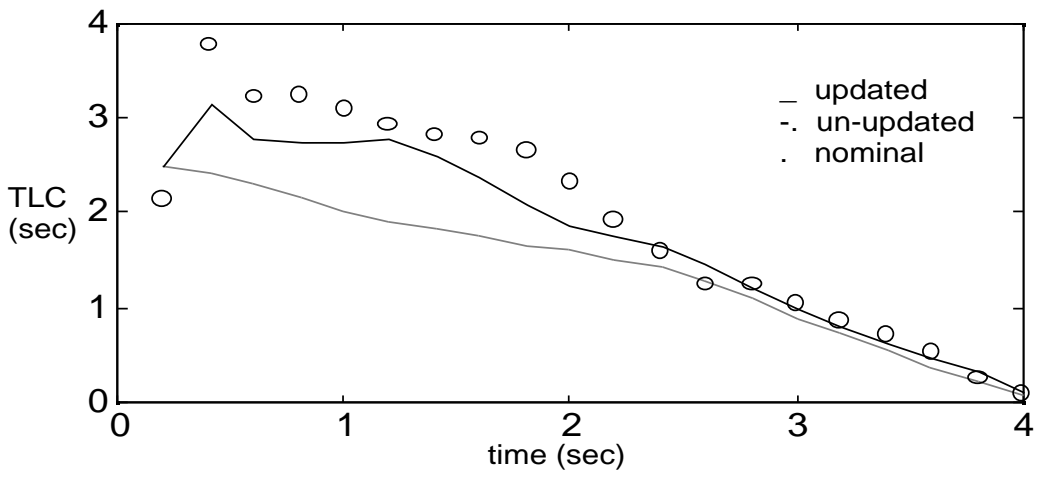

Fig. 11 Calculated TLC (curved road, 0.05 rad gradient)

The effect of road super-elevation is presented in Fig. 12. The TLC of the updated case is again closer to the nominal value than the un-updated case. The rms values of TLC error are 0.037 and 0.21 seconds. The TLC errors are reduced significantly compared to those of Fig. 8. This is because when the road has a proper super-elevation the tire forces generated by the steering will be reduced, i.e. the tire slip angles will be reduced. Since the cornering stiffness will be insensitive to road surface conditions when the slip angle is small, the TLC from the un-updated case is closer to the nominal value.

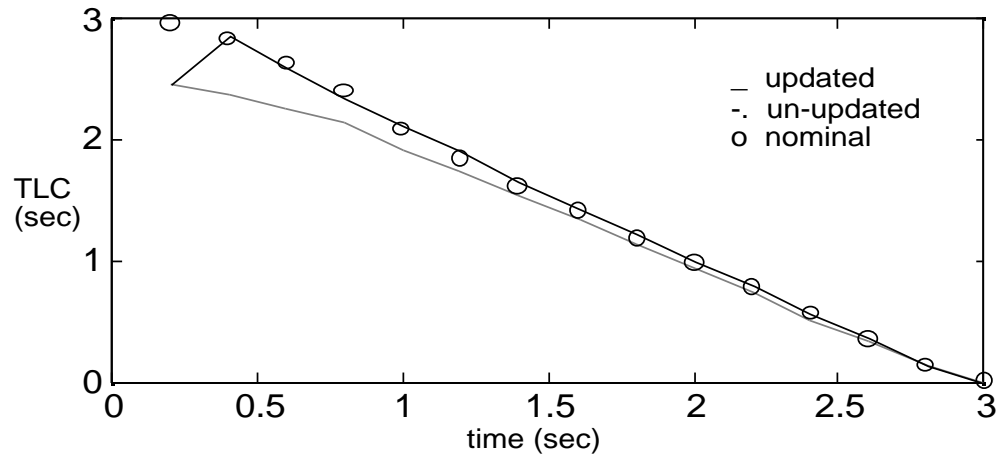

Fig. 12 Calculated TLC (Curved Road, 0.05 rad Super-elevation) 
When the vehicle is steered by sinusoidal input (a rough approximation of a drunk driver), the TLC was only slightly improved in a certain region which can be viewed more clearly in Fig. 14. The main reason of this dismal improvement is because the estimation scheme is sluggish in tracking varying signals. In this simulation the steering input is sinusoidal which means that the effective cornering stiffness changes continuously. The TLC calculation is improved more when the steering angle is large, i.e., when the cornering stiffness is not fast varying and effective cornering stiffness is small.

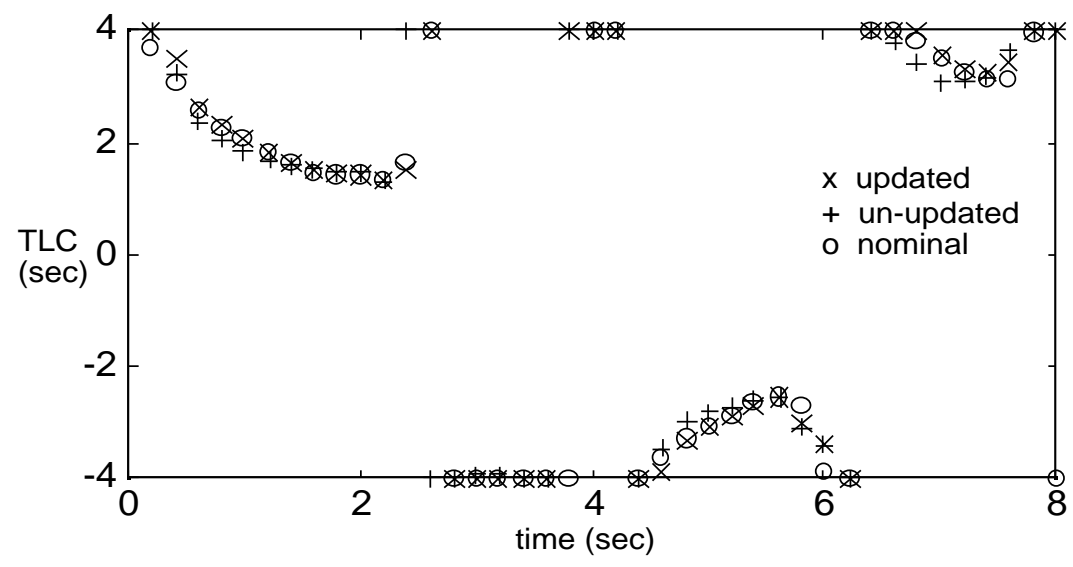

Fig. 13 Calculated TLC (straight road, sinusoidal steering angle input)

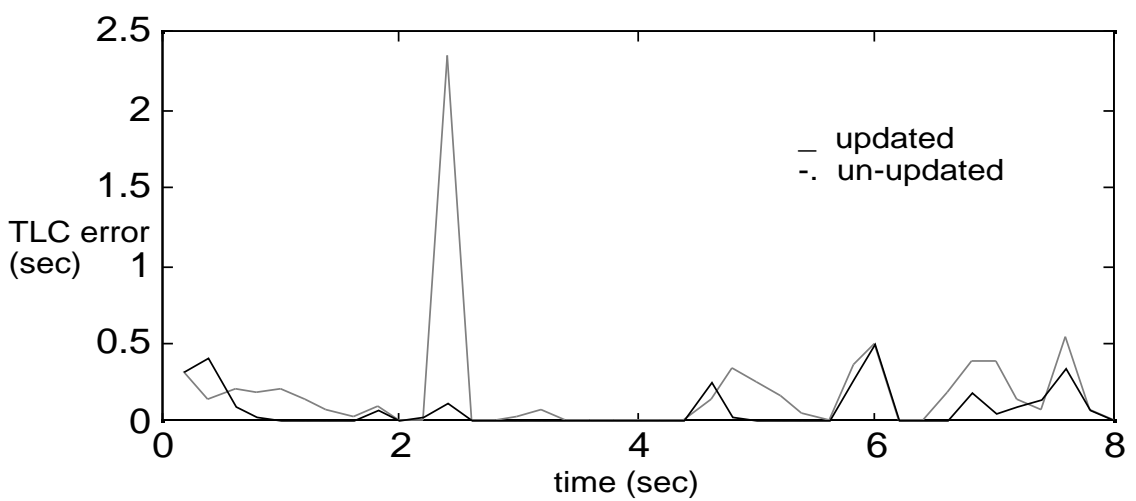

Fig. 14 TLC Error (straight road, sinusoidal steering angle input)

\section{CONCLUSIONS}

Both methods of modified adaptive observer and least square algorithm work well to estimate the road surface condition. The purpose of using the modified adaptive observer is that it can estimate the time variant nonlinear input without a mathematical form of the input. The effect of the vehicle parameters (tire cornering stiffness) on the TLC calculation is the main performance evaluation metric in this paper. It is found out that the accuracy of the TLC can be improved up to 0.5 second if the road surface condition is considered, but for some cases the accuracy of the TLC calculation of the updated one is still not good enough even though it has better results than that of the un-updated one. The solution is that we should modify the scheme of the vehicle lateral speed estimation. We may introduce a higher order vehicle model or use a disturbance rejection observer to increase the accuracy of the vehicle lateral speed estimation. It is left for the further investigation. It is also found that the TLC calculation error of the case that the cornering stiffness isn't updated will be reduced if the road has moderate super-elevation. For the case of sinusoidal steering input, the updated one can only have better performance on some regions. How 
Vehicle System Dynamics, Vol.25 Suppl., 1996, pp.413-425

to increase the response and accuracy of the cornering stiffness estimation is another challenge task.

\section{ACKNOWLEDGMENT}

The work is supported by the ITS Research Center of Excellence at the University of Michigan. The authors also wish to thank Prof. A.G. Ulsoy and other CAPC team members--Bob Ervin, , Chiu-Feng Lin and Paul Venhovens for their support.

\section{REFERENCES}

1. Lin, C.-F. and Ulsoy, A.G., "Calculation Of The Time To Lane Crossing And Analysis Of Its Frequency Distribution," American Control Conference, 1995.

2. Dieckmann, T., "Assessment of Road Grip by way of Measured Wheel Variables," Proceedings FISITA, London , June 1992.

3. Eichhorn, U. and Roth, J.,"Prediction And Monitoring of Tyre/Road Friction," Proceedings FISITA, London , June 1992.

4. Ito, M., Yoshioka, K. and Saji, T., "Estimation of Road Surface Conditions Using Wheel Speed Behavior," SAE no. 9438826 .

5. Pal, C., Hagiwara, I., Morishita, S. and Inoue, H., "Application of Neural Networks in Real Time Identification of Dynamic Structural Response and Prediction of Road-Friction Coefficient $\mu$ from Steady State Automobile Response," SAE no. 9438817

6. Pasterkamp, W. R. and Pacejka, H. B., "On Line Estimation of Tyre Characteristics for Vehicle Control," American Control Conference, 1995

7. Pacejka, H. B. and Sharp, R. S., "Shear Force Development by Pneumatic Tyres in Steady State Conditions: A Review of Modeling Aspects, "Vehicle System Dynamics, Vol. 20, 1991, pp. 121-176.

8. Mendel, J. M., "Lessons in Estimation Theory for Signal Processing, Communication, and Control, " Prentice Hall, Inc., Englewood Hill, New Jersey, 1995.

9. Messner, W., and Horowitz, R., "Identification of a Nonlinear Function in a Dynamical System," ASME Journal of Dynamic Systems, Measurement, and Control, Dec. 1993, Vol. 115, pp. 587-591.

10. Gillespie, T. D., "Fundamentals of vehicle dynamics," Warrendale, PA : Society of Automotive Engineers, 1992.

11. Venhovens P. J. Th., "Optimal Control of Vehicle Suspensions," Ph.D. Thesis, Delft University of Technology, Dutch, 1993.

12. Gustafsson F., "Slip-Based Estimation of Tire-Road Friction" , Submitted to the IEEE Transactions on Control Systems Technology. 